\title{
A CHARACTERIZATION OF A SPECIAL ORDERING IN A ROOT SYSTEM
}

\author{
PAOLO PAPI
}

(Communicated by Ronald M. Solomon)

\begin{abstract}
We give necessary and sufficient conditions for an ordering of a set of positive roots in a root system $R$ to be associated to a reduced expression of an element of the Weyl group of $R$. Finally we characterize the sets of positive roots which can be given such an ordering.
\end{abstract}

\section{INTRODUCTION}

Let $R$ be a reduced root system (see [3] for definitions) and $W$ the associated Weyl group. Let $\Pi=\left\{\alpha_{1}, \ldots, \alpha_{n}\right\}$ be a set of simple roots, and let $R^{+}, R^{-}$be respectively the sets of positive and negative roots corresponding to $\Pi$ so that we have $R=R^{+} \cup R^{-}, R^{-}=-R^{+}$. Finally let $s_{1}, \ldots, s_{n}$ be the reflections associated to the simple roots.

Fix a reduced expression of $w \in W$, say $s_{i_{1}} \cdots s_{i_{m}}$, and define

$$
\beta_{1}:=\alpha_{i_{1}} ; \quad \beta_{h}:=s_{i_{1}} \cdots s_{i_{h-1}}\left(\alpha_{i_{h}}\right), \quad h=2, \ldots, m .
$$

It is known (see [2]) that $\beta_{i} \neq \beta_{j}$ for $i \neq j$ and that $\left\{\beta_{1}, \ldots, \beta_{m}\right\} \subseteq R^{+}$. So a total ordering, which we denote by $<$, is induced on $\left\{\beta_{1}, \ldots, \beta_{m}\right\}$. If, in particular, we take $w$ to be the longest element in $W$, we obtain a total ordering in $R^{+}$.

This fact is useful in many contexts: for instance, in theory of quantum groups, this ordering is needed to define, through Lusztig's automorphisms (see [6]), the $q$-analogs of root vectors in a finite-dimensional semisimple Lie algebra having $R$ as associated root system. In this paper we give a simple combinatorial characterization of these types of ordering and some related results. We finally notice that some of our results can be given in the general framework of Coxeter groups.

\section{STATEMENT AND PROOF OF THE CRITERION}

We begin with a definition:

Definition. If $L=\left\{\gamma_{1}, \ldots, \gamma_{m}\right\}$ is an ordered set of positive roots, we say that $L$ is associated to $w \in W$ if there is a reduced expression $s_{i_{1}} \cdots s_{i_{m}}$ of $w$ such

Received by the editors February 4, 1992 and, in revised form, June 8, 1992.

1991 Mathematics Subject Classification. Primary 20F55; Secondary 17B20.

Key words and phrases. Ordering, root system, Coxeter group ,Weyl group. 
that

$$
\gamma_{1}=\alpha_{i_{1}} ; \quad \gamma_{h}=s_{i_{1}} \cdots s_{i_{h-1}}\left(\alpha_{i_{h}}\right), \quad h=2, \ldots, m
$$

The following results are well known (see, for instance, [1]).

Proposition. If $L$ is an ordered subset of $R^{+}$associated to $w \in W$, then as a set $L=R_{w^{-1}}$, where $R_{w^{-1}}:=\left\{\alpha \in R^{+}: w^{-1}(\alpha) \in R^{-}\right\}$. Consequently the length of $w$ equals the cardinaiity of $R_{w^{-1}}$.

Corollary. If $L, L^{\prime}$ are ordered subsets of $R^{+}$associated to the same $w \in W$, then $L$ and $L^{\prime}$ differ at most by the ordering.

Now we can state the main result.

Theorem. An ordered subset $L$ of $R^{+}$is associated to $w$ (for some $w \in W$ ) if and only if the following conditions are verified:

(1) If $\lambda, \mu \in L, \lambda<\mu$, and $\lambda+\mu \in R$, then $\lambda+\mu \in L$ and $\lambda<\lambda+\mu<\mu$.

(2) If $\lambda+\mu \in L, \lambda, \mu \in R^{+}$, then $\lambda$ or $\mu$ (or both) belong to $L$ and one of them precedes $\lambda+\mu$.

Remark. Condition (2) is not needed (since it is automatically verified) if we want to describe the orderings of $R^{+}$induced by a reduced expression of the longest element in $W$.

Proof. Suppose first that $L$ is associated to $s_{i_{1}} \cdots s_{i_{m}}=w$. Given $\lambda, \mu \in L$, we assume that $\lambda=s_{i_{1}} \cdots s_{i_{h-1}}\left(\alpha_{i_{h}}\right)$ and $\mu=s_{i_{1}} \cdots s_{i_{k-1}}\left(\alpha_{i_{k}}\right)$ with $1 \leq h<k \leq m$.

We check conditions (1) and (2). As regards (1), we have, by the proposition, $\lambda, \mu \in R_{w^{-1}}$, and, since $\lambda+\mu \in R$, we deduce that $\lambda+\mu \in L$; furthermore, we have $\lambda<\lambda+\mu<\mu$. To prove this assertion, we must show that it can be neither $\lambda<\mu<\lambda+\mu$ nor $\lambda+\mu<\lambda<\mu$.

If $\lambda+\mu=s_{i_{1}} \cdots s_{i_{t-1}}\left(\alpha_{i_{t}}\right)$ we define

$$
v:=s_{i_{1}} \cdots s_{i_{t}}, \quad u:=s_{i_{1}} \cdots s_{i_{k}}
$$

obtaining in the first case $\lambda+\mu \notin R_{u^{-1}}, \lambda, \mu \in R_{u^{-1}}$, which leads to a contradiction, and in the second case $\lambda+\mu \in R_{v^{-1}}, \lambda, \mu \notin R_{v^{-1}}$, which is absurd too; so condition (1) is fulfilled.

To prove the first statement of (2) suppose that $\lambda, \mu \notin L$. Then we have $\lambda, \mu \notin R_{w^{-1}}, \lambda+\mu \in R_{w^{-1}}$, which is a contradiction.

With an argument analogous to the previous one we can show that one (and only one by virtue of (1)) of the relations $\lambda<\lambda+\mu, \mu<\lambda+\mu$ holds, and the proof of $(2)$ is completed. To prove the "only if" part, we proceed by induction on the number of elements of $L=\left\{\beta_{1}, \ldots, \beta_{m}\right\}$. If $m=1$, we have to show that, if $L=\{\alpha\}$ verifies (1) and (2), then $\alpha$ is necessarily a simple root; but this is quite obvious recalling that simple roots are characterized as the indecomposable ones (those that cannot be written as a sum of two positive roots). If $\alpha$ is not simple, then there exist $\lambda, \mu \in R^{+}$such that $\alpha=\lambda+\mu$, and condition (2) implies $\lambda<\alpha$ or $\mu<\alpha$, a contradiction.

Now we notice that all subsets of $L$ of the form $\left\{\beta_{1}, \ldots, \beta_{j}\right\}, 1 \leq j \leq m$, verify (1) and (2). So we can apply induction to $\left\{\beta_{1}, \ldots, \beta_{m-1}\right\}$ obtaining a reduced expression $s_{i_{1}} \cdots s_{i_{m-1}}$ of an element $w^{\prime} \in W$ such that

$$
\beta_{1}=\alpha_{i_{1}} ; \quad \beta_{h}=s_{i_{1}} \cdots s_{i_{h-1}}\left(\alpha_{i_{h}}\right), \quad h=2, \ldots, m-1 .
$$

By the proposition, $R_{w^{\prime-1}}=\left\{\beta_{1}, \ldots, \beta_{m-1}\right\}$. 
We want to show that there is an index $i_{m}$ such that $w^{\prime}\left(\alpha_{i_{m}}\right)=\beta_{m}$; if we can do this, we have the assertion since $s_{i_{1}} \cdots s_{i_{m}}$ is a reduced expression of $w:=w^{\prime} s_{i_{m}} \in W$ to which $L$ is associated. (Notice that $s_{i_{1}} \cdots s_{i_{m}}$ is certainly a reduced expression since $w^{\prime}\left(\alpha_{i_{m}}\right) \in R^{+}$implies $l(w)=l\left(w^{\prime}\right)+1$.)

We have to prove that $w^{-1}\left(\beta_{m}\right)$ is a simple root; otherwise, it is decomposable, and so there exist $\lambda, \mu \in R^{+}$such that $w^{\prime-1}\left(\beta_{m}\right)=\lambda+\mu$, or $\beta_{m}=\lambda^{\prime}+\mu^{\prime}$ if we set $\lambda^{\prime}=w^{\prime}(\lambda), \mu^{\prime}=w^{\prime}(\mu)$. Now we first suppose that both $\lambda^{\prime}$ and $\mu^{\prime}$ are positive roots. Then (2) implies $\lambda^{\prime}<\beta_{m}$ or $\mu^{\prime}<\beta_{m}$. In the first case we have $\lambda^{\prime} \in R_{w^{\prime-1}}$, that is, $w^{\prime-1}\left(\lambda^{\prime}\right)=\lambda \in R^{-}$, which is absurd, and in the second one we have an analogous contradiction.

Since a positive root cannot be sum of two negative ones, the only further possibility that must be considered is $\lambda^{\prime} \in R^{+}, \mu^{\prime} \in R^{-}$. If we set $\mu^{\prime \prime}:=-\mu^{\prime}$, we have $\mu^{\prime \prime} \in R^{+}$and $\beta_{m}=\lambda^{\prime}-\mu^{\prime \prime}$. We claim that $\mu^{\prime \prime} \notin L$; otherwise, $\mu^{\prime \prime}<\beta_{m}$ and condition (1) forces $\mu^{\prime \prime}+\beta_{m}=\lambda^{\prime} \in L$ and $\lambda^{\prime}<\beta_{m}$, that is, $\lambda^{\prime} \in R_{w^{\prime-1}}$. As above, this leads to

$$
w^{\prime-1}\left(\lambda^{\prime}\right)=w^{\prime-1}\left(w^{\prime}(\lambda)\right)=\lambda \in R^{-},
$$

a contradiction. But if $\mu^{\prime \prime} \notin L$, then $\mu^{\prime \prime} \notin R_{w^{\prime-1}}$ and we have

$$
w^{\prime-1}\left(\mu^{\prime \prime}\right)=w^{\prime-1}\left(-\mu^{\prime}\right)=w^{\prime-1}\left(w^{\prime}(-\mu)\right)=-\mu \in R^{+},
$$

so we have the desired contradiction in this case too.

Corollary. There exists a one-to-one correspondence between reduced expressions of elements $w \in W$ and orderings of the set $R_{w^{-1}}$ satisfying conditions (1) and (2).

Such orderings will be called compatible.

\section{SOME RELATED RESULTS}

Now we will find the conditions under which any subset $L \subseteq R^{+}$can be given a compatible ordering, so that $L$ is associated to some $w \in W$-we will actually see that $L$ is associated to a unique $w-$.

Theorem. $L \subseteq R^{+}$can be given a compatible ordering if and only if it satisfies the following properties:

(I) If $\lambda, \mu \in L$ and $\lambda+\mu \in R$, then $\lambda+\mu \in L$.

(II) If $\lambda+\mu \in L, \lambda, \mu \in R^{+}$, then $\lambda \in L$ or $\mu \in L$.

Moreover, any such set is associated to a unique $w \in W$.

Proof. We notice preliminarily that the theorem can be formulated as follows: conditions (I) and (II) are necessary and sufficient for $L \subseteq R^{+}$to be of the form $R_{w}$ (and such $w$ is unique). By the previous theorem, conditions (I) and (II) are obviously necessary. Suppose now that $L=\left\{r_{1}, \ldots, r_{n}\right\} \subseteq R^{+}$satisfies (I) and (II). We will prove simultaneously the existence of a compatible ordering and the uniqueness of the associated element of $W$ by induction on $n$.

It is clear, by (II), that a simple root, say $\alpha$, belongs to $L$; this remark proves the assertion if $n=1$. Now we assume the statement for $n-1$. Let $s \equiv s_{\alpha}$ and $L^{\prime}$ denote respectively the fundamental reflection relative to $\alpha$ and the set $s(L-\{\alpha\})$. It is well known that $L^{\prime} \subseteq R^{+}-\{\alpha\}$; moreover, a simple check 
shows that $L^{\prime}$ still verifies (I) and (II). In fact, if $\lambda^{\prime}, \mu^{\prime} \in L^{\prime}$ and their sum is a root, then $\lambda^{\prime}=s(\lambda), \mu^{\prime}=s(\mu)$ with $\lambda, \mu \in L-\{\alpha\}$ so that $\lambda^{\prime}+\mu^{\prime}=s(\lambda+\mu)$; therefore, $\lambda+\mu \in R$ and hence $\lambda+\mu \in L$ by (I) and in turn $\lambda^{\prime}+\mu^{\prime} \in L^{\prime}$.

To show that (II) holds in $L^{\prime}$, suppose $\lambda^{\prime}+\mu^{\prime} \in L^{\prime}, \lambda^{\prime}, \mu^{\prime} \in R^{+}$. Then $\lambda^{\prime}+\mu^{\prime}=s(\xi)$ with $\xi \in L-\{\alpha\}$. We have $\xi=s\left(\lambda^{\prime}\right)+s\left(\mu^{\prime}\right)$. If now $s\left(\lambda^{\prime}\right), s\left(\mu^{\prime}\right) \in$ $R^{+}$, applying (II) to $L$ we obtain that either $s\left(\lambda^{\prime}\right)$ or $s\left(\mu^{\prime}\right)$ belongs to $L$ and consequently $\lambda^{\prime}$ or $\mu^{\prime}$ belongs to $L^{\prime}$. If instead $\lambda^{\prime}=\alpha$ then $\xi+\alpha=s\left(\mu^{\prime}\right)$ and, therefore, applying (I) to $\xi \in L$ and $\alpha \in L$, we can deduce $s\left(\mu^{\prime}\right) \in L$, that is, $\mu^{\prime} \in L^{\prime}$. So by induction there exists a unique $w \in W$ to which $L^{\prime}$ is associated. We claim that $L$ is associated to $s w$; we first notice that, since $\alpha \notin L^{\prime}, w^{-1}(\alpha) \in R^{+}$so that $l(s w)=l\left(w^{-1} s\right)=l\left(w^{-1}\right)+1=l(w)+1=n$ and $\left|R_{w^{-1} s}\right|=n$.

Now, if $\xi \in L-\{\alpha\}$, then $s(\xi) \in L^{\prime}$ and $(s w)^{-1}(\xi)=w^{-1} s(\xi) \in R^{-}$by induction; moreover, $(s w)^{-1}(\alpha)=-w^{-1}(\alpha) \in R^{-}$so that $L \subseteq R_{w^{-1} s}$. But $|L|=\left|R_{w^{-1} s}\right|=n$; therefore, $L=R_{w^{-1} s}$ as we wanted.

Suppose finally that $L$ is associated to $w^{\prime} \in W$ too. Then we have

$$
R_{w^{-1}}=L^{\prime}=s(L-\{\alpha\})=s\left(R_{w^{\prime-1}}-\{\alpha\}\right)=R_{w^{\prime-1} s}
$$

and by induction $w^{-1}=w^{\prime-1} s$ so that $w^{\prime}=s w$.

Remark. As we noticed in the introduction, both theorems work for any Coxeter group $W$. More precisely, we consider a Coxeter system $(W, S)$ and its geometric representation $\sigma: W \rightarrow \mathrm{GL}(V)$, where $V$ is a real vector space having a basis $\left\{\alpha_{s}: s \in S\right\}$ in one-to-one correspondence with $S$ (for definitions and details see [4]). Now we have only to remark that all notions and results used in the previous proofs still hold in the general case, with the only exception being the following property, which must be assumed: the only indecomposable roots in $R^{+}$are the simple ones. Obviously, all subsets of $R^{+}$must be taken finite; in particular, the remark after the first theorem is meaningless if $R^{+}$is not finite.

We finally describe a combinatorial way to pass from a compatible ordering on $L \subseteq R^{+}$to another one. Let $L$ be a subset of $R^{+}$verifying (I) and (II), and let $\mathscr{S}$ denote the set of all compatible orderings on $L$.

Definition. Suppose that both the ordering $S_{1}=\{\cdots \lambda \mu \cdots\}$ and the ordering $S_{2}=\{\cdots \mu \lambda \cdots\}$ belong to $\mathscr{S}$; this notation means:

$$
\begin{aligned}
& S_{1}=\left\{r_{i_{1}}, r_{i_{2}}, \ldots, r_{i_{n}}\right\} ; \quad S_{2}=\left\{r_{i_{1}^{\prime}}, r_{i_{2}^{\prime}}, \ldots, r_{i_{n}^{\prime}}\right\} ; \\
& r_{i_{h}}=r_{i_{h}^{\prime}}, \quad h=1, \ldots, j-1, j+2, \ldots, n ; \\
& r_{i_{j}}=r_{i_{j+1}^{\prime}}=\lambda ; \quad r_{i_{j+1}}=r_{i_{j}^{\prime}}=\mu .
\end{aligned}
$$

Then we say that passing from $S_{1}$ to $S_{2}$ (or vice-versa) realizes an elementary move of type 0 (at the jth place) on $S_{1}$ (resp. $S_{2}$ ). Analogously, if

$$
\begin{aligned}
& S_{1}=\{\cdots \lambda \lambda+\mu \mu \ldots\} \in \mathscr{S}, \quad S_{2}=\{\cdots \mu \lambda+\mu \lambda \cdots\} \in \mathscr{S} \\
& S_{1}=\{\cdots \lambda \lambda+\mu \lambda+2 \mu \mu \cdots\} \in \mathscr{S}, \quad S_{2}=\{\cdots \mu \lambda+2 \mu \lambda+\mu \lambda \cdots\} \in \mathscr{S} \text {; } \\
& S_{1}=\left\{\lambda \lambda+\mu 2 \lambda+3 \mu \quad S_{2}=\{\mu \lambda+3 \mu \lambda+2 \mu\right. \\
& \lambda+2 \mu \lambda+3 \mu \mu\} \in \mathscr{S}, \quad 2 \lambda+3 \mu \lambda+\mu \mu\} \in \mathscr{S}
\end{aligned}
$$


then the corresponding move $S_{1} \rightarrow S_{2}$ (or $S_{2} \rightarrow S_{1}$ ) is called, respectively, an elementary move of type $1,2,3$.

Consider the braid relations:

$$
\begin{array}{lll}
0^{\prime} & s_{i_{j}} s_{i_{j+1}}=s_{i_{j+1}} s_{i_{j}} & \text { if } a_{i_{j} i_{j+1}} a_{i_{j+1} i_{j}}=0, \\
1^{\prime} & s_{i_{j}} s_{i_{j+1}} s_{i_{j}}=s_{i_{j+1}} s_{i_{j}} s_{i_{j+1}} & \text { if } a_{i_{j} i_{j+1}} a_{i_{j+1} i_{j}}=1, \\
2^{\prime} & s_{i_{j}} s_{i_{j+1}} s_{i_{j}} s_{i_{j+1}}=s_{i_{j+1}} s_{i_{j}} s_{i_{j+1}} s_{i_{j}} & \text { if } a_{i_{j} i_{j+1}} a_{i_{j+1} i_{j}}=2, \\
3^{\prime} & s_{i_{j}} s_{i_{j+1}} s_{i_{j}} s_{i_{j+1}} s_{i_{j}} s_{i_{j+1}}=s_{i_{j+1}} s_{i_{j}} s_{i_{j+1}} s_{i_{j}} s_{i_{j+1}} s_{i_{j}} & \text { if } a_{i_{j} i_{j+1}} a_{i_{j+1} i_{j}}=3
\end{array}
$$

(here $\left(a_{i j}\right)$ is the Cartan matrix of $R$; see [3]). Let $L=R_{w^{-1}}, \mathscr{S}$ be as above, and $S_{1}, S_{2} \in \mathscr{S}$ correspond to the reduced expressions of $w, s_{i_{1}} \cdots s_{i_{n}}$, $s_{i_{1}^{\prime}} \cdots s_{i_{n}^{\prime}}$ respectively. It is easy to see that an elementary move $S_{1} \rightarrow S_{2}$ of type $h, h \in\{0,1,2,3\}$, at the $j$ th place corresponds to apply a braid relation of type $h^{\prime}$ at the $j$ th fundamental reflection of $s_{i_{1}} \cdots s_{i_{n}}$ and vice-versa.

Recall now the following well-known result of Iwahori (see [5]): if $W$ is a Weyl group and $w \in W$, different reduced expressions of $w$ can be transformed one into another using only the braid relations. It is clear that this theorem can be reformulated in the language of compatible orderings as follows.

Theorem. If $S, S^{\prime}$ are two compatible orderings on $R_{w}, w \in W$, one can pass from $S$ to $S^{\prime}$ through a finite sequence of elementary moves.

\section{ACKNOWLEDGMENT}

I would like to express my sincere thanks to Professor Claudio Procesi for his helpful suggestions.

\section{REFERENCES}

1. N. Bourbaki, Groupes et algebres de Lie, Chapitres 4-6, Hermann, Paris, 1968.

2. H. Hiller, Geometry of Coxeter groups, Pitman Res. Notes Math. Ser., vol. 54, Longman Sci. Tech., Harlow, 1982.

3. J. E. Humphreys, Introduction to Lie algebras and representation theory, Graduate Texts in Math., vol. 9, Springer-Verlag, New York, 1972.

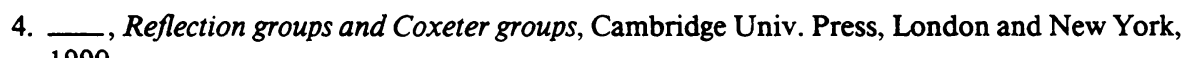
1990.

5. N. Iwahori, On the structure of a Hecke ring of a Chevalley group over a finite field, J. Fac. Sci. Univ. Tokyo Sect. IA Math. 10 (1964), 215-236.

6. G. Lusztig, Quantum groups at roots of one, Geom. Dedicata 35 (1990), 89-114.

Dipartimento di Matematica, Università di Pisa, Via F. Buonarroti 2, 56127 Pisa, Italy E-mail address: papi@dm.unipi.it 